Ruth V. Sabariego was born in Vigo, Spain, on April 21, 1974. She received the M.Sc. degree in telecommunication engineering from the University of Vigo, Spain, in 1998. She is currently a Ph.D. student at the Dept. of Applied Electricity (ELAP), University of Liège, Belgium. Her research interest include numerical techniques for computational electromagnetics, applied mathematics, radiation hazards and bio-electromagnetics.

Johan Gyselinck was born in Ghent, Belgium, on July 3, 1967. He graduated in Electrical and Mechanical Engineering at the Ghent University in 1991 and received the Ph.D. degree from the same university in 2000. Since 2000 he is a Postdoctoral Researcher at the Department of Applied Electricity, University of Liège, Belgium. His research mainly deals with the numerical modelling of coupled problems in electromagnetic systems, in particular the finite element analysis of electrical machines.

Christophe Geuzaine is a Postdoctoral Researcher with the Belgian National Fund for Scientific Research. He is currently working at Caltech's Applied and Computational Mathematics Department on high-order, high-frequency methods for surface scattering problems.

Patrick Dular was born in Rocourt, Belgium, on August 28, 1967. He received the electrical engineering degree and Ph.D. degree in applied sciences from the University of Liége, Belgium, in 1990 and 1994, respectively. He is currently with the Department of Applied Electricity, University of Liège, as a Research Associate with the Belgian National Fund for Scientific Research (F.N.R.S.). His research deals mainly with the numerical modelling of coupled problems in electromagnetic systems, including both physical models and numerical techniques coupling.

Willy Legros was born in Dolembreux, Belgium, on December 24, 1940. He received the M.Sc. and Ph.D. degrees in electrical engineering from the University of Liège, Belgium, in 1963 and 1970 respectively. He is professor at the aforementioned university since 1978. Currently, he is the head of the Department of Applied Electricity and the rector of the University of Liège. 


\title{
Application of the Fast Multipole Method to the 2D Finite Element-Boundary Element Analysis of Electromechanical Devices
}

\author{
R. V. Sabariego, J. Gyselinck, C. Geuzaine, P. Dular and W. Legros \\ Department of Electrical Engineering, Institut Montefiore, University of Liège, \\ Sart Tilman Campus, building B28,B-4000 Liège, Belgium, e-mail: R.Sabariego@ulg.ac.be
}

\begin{abstract}
The present paper deals with the fast multipole acceleration of the $2 D$ finite element-boundary element modelling of electromechanical devices. It is shown that the fast multipole method, usually applied to large $3 D$ problems, can also lead to a reduction in computational time when dealing with relatively small $2 D$ problems, provided that an adaptive truncation scheme for the expansion of the 2D Laplace Green function is used. As an application example, the $2 D$ hybrid modelling of a linear actuator is studied, taking into account saturation, the voltage supply and the mechanical equation. The computational cost without and with fast multipole acceleration is discussed for both the linear and nonlinear case.
\end{abstract}

Keywords Fast multipole method, Finite element method, Boundary element method, Hybrid method, Electromechanical devices

\section{Introduction}

Hybrid finite element - boundary element (FE-BE) models are particularly suited for motional nonlinear magnetic field problems (Henrotte, 1994; Nysveen, 1997; Fetzer, 1999). The finite element (FE) method easily accounts for saturable and conducting media, while the boundary element (BE) method provides a rigorous treatment for open problems and allows to consider movement without any tedious mesh manipulations (remeshing or moving band definition). Nevertheless, the BE part of the hybrid FE-BE method generates dense blocks in the system matrix.

The fast multipole method (FMM) (Rokhlin, 1983) can be usefully applied to the BE part in order to reduce the computational cost of the hybrid FE-BE model. It is used in conjunction with an iterative solver, e.g. GMRES (Saad, 1986), diminishing the storage requirements and speeding up the matrixvector multiplication in every iteration. It also brings about a less costly assembly of the BE blocks in the system matrix of algebraic equations, which is particularly important for 2D problems (Sabariego, 2002).

In the domain of electromagnetic field computations, the FMM has been successfully applied to BE models in both high frequency (Burkholder, 1996) and low frequency (Buchau, 2000) problems. Hybrid FE-BE models have mainly been considered in scattering applications (Lu, 1996).

This paper deals with the application of the FMM to the hybrid FE-BE resolution of 2D electromechanical problems. The first section outlines the hybrid modelling of a magnetostatic problem. The single-level FMM method is briefly described in the following section. Herein, an adaptive truncation scheme for the 2D Laplace Green function, proposed by the authors in (Sabariego, 2002), is used. Then, some aspects relative to the analysis of electromechanical devices by a hybrid FE-BE method taking into account the FMM are considered. This acceleration method has already been successfully applied to hybrid models, but, to the best of our knowledge, not yet to motional problems.

As an application example, the 2D modelling of a linear actuator is studied in detail. The transient phenomenon will be modelled taking into account the nonlinear behaviour of materials, the voltage supply and the mechanical equation. 


\section{Hybrid FE-BE model}

First we consider a linear magnetostatic problem in $\mathbb{R}^{2}$. The FE method is used in a domain $\Omega$ comprising all saturable parts of the model and a domain $\Omega_{s}$ in which the current density $\underline{j}=j_{s}(x, y, t) \underline{1}_{z}$, directed along the $z$-axis, is given. The $\mathrm{BE}$ method takes into account the free space exterior to $\Omega$, the boundary of which is denoted $\Gamma$. The FE part $\Omega$ and the BE part $\Gamma$ of the hybrid model are discretised with e.g. triangular elements and straight line segments respectively.

The governing differential equations and constitutive laws are

$$
\operatorname{curl} \underline{h}=\underline{j}, \quad \operatorname{div} \underline{b}=0 \quad \text { and } \quad \underline{h}=\nu \underline{b} \quad \text { in } \mathbb{R}^{2},
$$

where the $z$-component of the magnetic field $\underline{h}(x, y, t)$ and the magnetic induction $\underline{b}(x, y, t)$ vanish, and the magnetic reluctivity $\nu$ is constant.

Permanent magnets can be included in the analysis as well. The constitutive law $\underline{h}=\nu(\underline{b}-\underline{b})$, where $\underline{b}_{r}$ is the remanent induction, leads to an equivalent current density curl $\left(\nu \underline{b}_{r}\right)$ in the permanentmagnet domains and to a current layer on their boundary. In case of a uniform magnetisation (constant $\nu \underline{b}_{r}$ ), only the latter is nonzero.

The problem is formulated in terms of the magnetic vector potential $\underline{a}=a(x, y) \underline{1}_{z}$ in $\Omega$ and the equivalent current layer $\underline{q}=q(\xi) \underline{1}_{z}$ on $\Gamma$ (Geuzaine, 2001).

For any continuous potential $a,(1 \mathrm{~b})$ is fullfilled on account of

$$
\underline{b}=\operatorname{curl} \underline{a}=\underline{1}_{z} \times \operatorname{grad} a .
$$

From $\operatorname{curl} \underline{h}=-\operatorname{div}(\nu \operatorname{grad} a) \underline{1}_{z}$, it follows that the weak form of Ampère's law (1a) is given by

$$
\int_{\Omega} \nu \operatorname{grad} a \cdot \operatorname{grad} a^{\prime} \mathrm{d} \Omega=\int_{\Omega_{s}} j_{s} a^{\prime} \mathrm{d} \Omega+\oint_{\Gamma} \nu \frac{\partial a}{\partial n} a^{\prime} \mathrm{d} \Gamma
$$

where the test function $a^{\prime}$ is continuous in $\Omega$.

The coupling with the BE model is done through the contour integral in (3) of the product of the test function $a^{\prime}$ and the tangential component of the magnetic field on $\Gamma, h_{t}=\nu b_{t}=\nu \frac{\partial a}{\partial n}=\nu \underline{n} \cdot \operatorname{grad} a$, where $\underline{n}$ is the unit normal vector on $\Gamma$ pointing into $\Omega$. Indeed, the potential $a$ in $\mathbb{R}^{2} \backslash \Omega$ can be expressed in terms of the equivalent current layer $q$ on $\Gamma$ :

$$
a=\frac{1}{\nu_{0}} \oint_{\Gamma} q G \mathrm{~d} \Gamma \quad \text { with } \quad \mathrm{G}=-\frac{1}{2 \pi} \ln \mathrm{r}
$$

where the 2D Laplace Green function $G(r)$ depends on the distance $r$ between a source point (on $\Gamma$ ) and an observation point (in $\mathbb{R}^{2} \backslash \Omega$ ). Further, from (4a) it follows that the tangential magnetic field on the boundary $\Gamma$ is given by

$$
\nu \frac{\partial a}{\partial n}=\frac{1}{2} q+\oint_{\Gamma} q \frac{\partial G}{\partial n} \mathrm{~d} \Gamma
$$

On the basis of the discretisation of $\Omega$ and $\Gamma$, \#a basis functions $\alpha_{j}(x, y)$ and $\# q$ basis functions $\beta_{l}(\xi)$ are defined for the vector potential $a(x, y)$ and the equivalent current layer $q(\xi)$ respectively:

$$
a(x, y)=\sum_{j=1}^{\# a} a_{j} \alpha_{j}(x, y) \quad \text { and } \quad q(\xi)=\sum_{l=1}^{\# q} q_{l} \beta_{l}(\xi) .
$$

The coefficients $a_{j}$ and $q_{l}$ are assembled in the column matrices $\boldsymbol{A}$ and $\boldsymbol{Q}$ :

$$
\boldsymbol{A}=\left[\begin{array}{lll}
a_{1} & \ldots & a_{\# a}
\end{array}\right]^{T} \text { and } \boldsymbol{Q}=\left[\begin{array}{lll}
q_{1} & \ldots & q_{\# q}
\end{array}\right]^{T} .
$$

By employing the $\# a$ basis functions $\alpha_{i}(x, y)$ as test functions in the weak form (3) and considering (5), a system of \#a algebraic equations is obtained. Equation (4a) is weakly imposed by weighing it on 
$\Gamma$ with the $\# q$ basis functions $\beta_{k}(\xi)$. The resulting system of $\# a+\# q$ equations of the hybrid model can thus be written as:

$$
\left[\begin{array}{cc}
\boldsymbol{S} & \boldsymbol{C} \\
\boldsymbol{D}^{T} & \boldsymbol{M}
\end{array}\right]\left[\begin{array}{l}
\boldsymbol{A} \\
\boldsymbol{Q}
\end{array}\right]=\left[\begin{array}{l}
\boldsymbol{J} \\
0
\end{array}\right]
$$

where $\boldsymbol{S}$ is a sparse $\# a \times \# a$ FE matrix, $\boldsymbol{C}$ and $\boldsymbol{D}$ are partially dense $\# a \times \# q$ matrices and $\boldsymbol{M}$ is a full $\# q \times \# q$ BE matrix. Their elements are given by

$$
\begin{aligned}
S_{i, j} & =\oint_{\Omega} \nu \operatorname{grad} \alpha_{i} \cdot \operatorname{grad} \alpha_{j} \mathrm{~d} \Omega \\
C_{i, l} & =\oint_{\Gamma} \alpha_{i}\left(\frac{1}{2} \beta_{l}+\oint_{\Gamma} \beta_{l} \frac{\partial G}{\partial n} \mathrm{~d} \Gamma\right) \mathrm{d} \Gamma \\
D_{j, k} & =\oint_{\Gamma} \alpha_{j} \beta_{k} \mathrm{~d} \Gamma \quad \text { and } \\
M_{k, l} & =\frac{1}{\nu_{0}} \oint_{\Gamma} \beta_{k}\left(\oint_{\Gamma} \beta_{l} G \mathrm{~d} \Gamma\right) \mathrm{d} \Gamma .
\end{aligned}
$$

The \#a $\times 1$ column matrix $\boldsymbol{J}$ follows from the imposed current density in $\Omega_{s}$ :

$$
J_{i}=\int_{\Omega_{s}} j_{s} \alpha_{i} \mathrm{~d} \Omega
$$

Solving the problem requires the assembly of the system of algebraic equations (8) and its resolution. The assembly of the BE part is expensive, especially when numerical integration is used. If straight line elements and piecewise constant basis functions are used for $q(\xi, t)$, the inner integrals in (10) and (12) can be evaluated analytically.

\section{Fast multipole method}

The fast multipole method (FMM) reduces the operational count by spatially decomposing the boundary $\Gamma$ into \#g groups of elements, $\Gamma=\bigcup_{g=1}^{\# g} \Gamma_{g}$, and determining the interactions between distant groups by means of the multipole expansion of the Green function. Hereto, for each group a geometrical center is considered. This is illustrated in Figure 1.

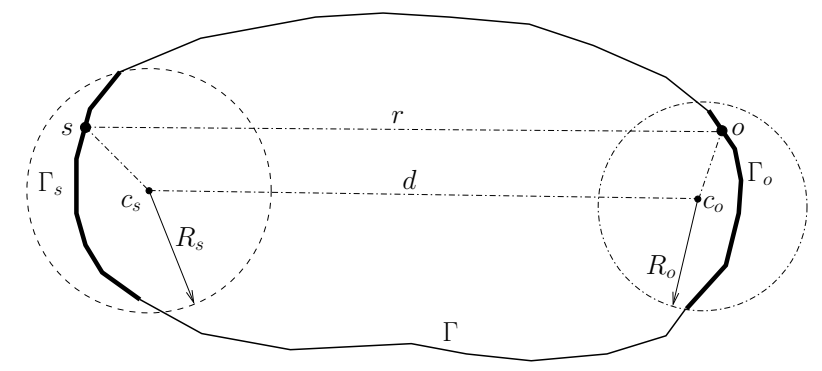

Figure 1. Distant groups $\Gamma_{s}$ and $\Gamma_{o}$ on the contour $\Gamma$, with respective centers $c_{s}$ and $c_{o}$

\section{Multipole expansion}

For sake of simplicity, points in $\mathbb{R}^{2}$ will be denoted by complex numbers, i.e. $\boldsymbol{z}=x+\imath y=r e^{\imath \phi}$. Let $\boldsymbol{z}_{s}$ be a source point in a given group centered in $\boldsymbol{z}_{s c}$ and $\boldsymbol{z}_{o}$ an observation point in a distant group of center $\boldsymbol{z}_{o c}$. Omitting the factor $-1 / 2 \pi$, the $2 \mathrm{D}$ Laplace Green function (4b) is then expanded as (Rokhlin, 1983):

$$
\ln r=\Re\left(\ln \left(\boldsymbol{z}_{o}-\boldsymbol{z}_{s}\right)\right)=\Re\left(\sum_{u=0}^{\infty} \sum_{v=0}^{\infty} \mathcal{D}_{u}\left(\boldsymbol{z}_{o c}, \boldsymbol{z}_{o}\right) \mathcal{T}_{u, v}\left(\boldsymbol{z}_{o c}, \boldsymbol{z}_{s c}\right) \mathcal{A}_{v}\left(\boldsymbol{z}_{s c}, \boldsymbol{z}_{s}\right)\right),
$$


with

$$
\begin{aligned}
\mathcal{D}_{u}\left(\boldsymbol{z}_{o c}, \boldsymbol{z}_{o}\right) & =\left(\boldsymbol{z}_{o c}-\boldsymbol{z}_{o}\right)^{u}, \\
\mathcal{T}_{u, v}\left(\boldsymbol{z}_{o c}, \boldsymbol{z}_{s c}\right) & =\left\{\begin{array}{lll}
\ln \left(\boldsymbol{z}_{o c}-\boldsymbol{z}_{s c}\right) & u=0 & \text { and } \quad v=0, \\
\frac{-(u+v-1) !}{u ! v !\left(\boldsymbol{z}_{o c}-\boldsymbol{z}_{s c}\right)^{u+v}} & u \neq 0 & \text { or } \quad v \neq 0,
\end{array}\right. \\
\mathcal{A}_{v}\left(\boldsymbol{z}_{s c}, \boldsymbol{z}_{s}\right) & =\left(\boldsymbol{z}_{s}-\boldsymbol{z}_{s c}\right)^{v} .
\end{aligned}
$$

In practice, the multipole expansion (14) must be truncated by considering $0 \leq u \leq p$ and $0 \leq v \leq p$, where the truncation number $p$ is sufficiently large to limit the error to a prescribed value $\varepsilon$ :

$$
\left|\ln r-\Re\left(\sum_{u=0}^{p} \sum_{v=0}^{p} \mathcal{D}_{u} \mathcal{T}_{u, v} \mathcal{A}_{v}\right)\right|<\varepsilon .
$$

In (Rokhlin, 1983), the truncation number is taken as $p=\log _{2}(1 / \varepsilon)$. In most cases, this expression overestimates the truncation number. Indeed, if the distance between the source point and its group center and the distance between the observation point and its group center are small compared to the distance $d$ between the two group centers, a smaller number of terms suffices.

A more economic law takes those distances into account. Let us denote by $R_{s}$ the maximum distance between a source point in a source group and its center, and by $R_{o}$ the maximum distance between an observation point in an observation group and its center (see Figure 1). The value of $p$ as a function of $R_{o} / d$ and $R_{s} / d$ for $\varepsilon=10^{-6}$ is depicted in Figure 2. It can be seen that $p=\log _{2}(1 / \varepsilon)$ corresponds to $R_{o} / d=R_{s} / d=0.35$.

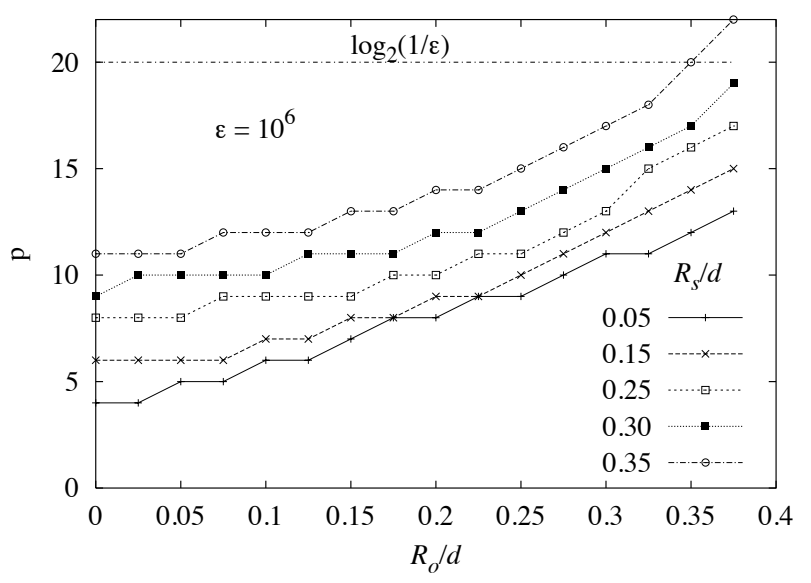

Figure 2. Truncation number $p$ as a function of $R_{o} / d$ and $R_{s} / d$ for $\varepsilon=10^{-6}$

In order to apply the FMM to (10), the expansion of $\operatorname{grad} G$ is necessary as well. It can be straightforwardly obtained by deriving (15) with respect to the coordinates of the observation point. The process to follow is then analogous.

\section{Application of the FMM}

Two groups $\Gamma_{s}$ and $\Gamma_{o}$ are said to be 'far' groups if $R_{s} / d<\tau$ and $R_{o} / d<\tau$, where $d$ is the distance between the group centers and where $\tau$ is chosen smaller than $1 / 2$.

The approximation of the matrix $\boldsymbol{M}$ can be formally written as

$$
\boldsymbol{M} \approx \boldsymbol{M}^{\text {near }}+\boldsymbol{M}^{f a r}=\boldsymbol{M}^{\text {near }}+\underbrace{\sum_{o=1}^{\# g} \sum_{s=1}^{\# g}}_{\Gamma_{o}, \Gamma_{s} \text { far }} \boldsymbol{M}_{o, s}^{f a r}
$$


and analogously for the part of matrix $\boldsymbol{C}$ that depends on $\frac{\partial G}{\partial n}$.

Let us consider the degrees of freedom $q_{k}$ and $q_{l}$ of $q(\xi)$ with associated basis functions $\beta_{k}(\xi)$ and $\beta_{l}(\xi)$ that are nonzero on the respective far groups $\Gamma_{o}$ and $\Gamma_{s}$. Substituting (14) in (12), the contribution to the corresponding element in $\boldsymbol{M}^{f a r}$ is given by

$$
\left(\boldsymbol{M}_{o, s}^{f a r}\right)_{k, l}=\Re\left(\sum_{u=0}^{p} \boldsymbol{M}_{o, k, u}^{\mathcal{D}} \sum_{v=0}^{p} \boldsymbol{M}_{u, v}^{\mathcal{T}} \boldsymbol{M}_{s, l, v}^{\mathcal{A}}\right),
$$

with

$$
\begin{aligned}
\boldsymbol{M}_{o, k, u}^{\mathcal{D}} & =\int_{\Gamma_{o}} \beta_{k} \mathcal{D}_{u}\left(\boldsymbol{z}_{c_{o}}, \boldsymbol{z}\right) \mathrm{d} \Gamma \\
\boldsymbol{M}_{u, v}^{\mathcal{T}} & =-\frac{1}{2 \pi \nu_{0}} \mathcal{T}_{u, v}\left(\boldsymbol{z}_{c_{o}}, \boldsymbol{z}_{c_{s}}\right) \quad \text { and } \\
\boldsymbol{M}_{s, l, v}^{\mathcal{A}} & =\int_{\Gamma_{s}} \beta_{l} \mathcal{A}_{v}\left(\boldsymbol{z}_{c_{s}}, \boldsymbol{z}\right) \mathrm{d} \Gamma .
\end{aligned}
$$

In case of straight line elements and piecewise constant basis functions, the integrals in (21-23) can be evaluated analytically considering

$$
\int_{z_{1}}^{z_{2}}\left(\boldsymbol{z}_{c}-\boldsymbol{z}\right)^{u} \mathrm{~d} \boldsymbol{z}=\frac{\left(\boldsymbol{z}_{c}-\boldsymbol{z}_{1}\right)^{u+1}-\left(\boldsymbol{z}_{c}-\boldsymbol{z}_{2}\right)^{u+1}}{u+1} .
$$

The aim of the formal decomposition (20) is speeding up the multiplication of $\boldsymbol{M}^{\text {far }}$ by a trial vector $\boldsymbol{Q}$, required for the iterative solution of the system of algebraic equations (8). Group by group, the field produced by the current layer in the considered group is aggregated into its center by (23). This aggregated field is then subsequently translated to the centers of all the far groups by (22), and finally the aggregated and translated field is disaggregated into the degrees of freedom of the far groups thanks to (21). The multiplication $\boldsymbol{M}^{\text {far }} \boldsymbol{Q}$ is further accelerated by means of the adaptive truncation scheme following the law $p=p\left(R_{s} / d, R_{o} / d, \varepsilon\right)$ shown in Figure 2 .

The assembly stage of the FMM consists in calculating and storing the required complex numbers $\boldsymbol{M}_{o, k, u}^{\mathcal{D}}, \boldsymbol{M}_{u, v}^{\mathcal{T}}$ and $\boldsymbol{M}_{s, l, v}^{\mathcal{A}}$. The matrix $\boldsymbol{M}^{f a r}$ itself is never built. The matrix $\boldsymbol{M}^{\text {near }}$ is calculated in the conventional way and stored using a sparse storage scheme. For the $\boldsymbol{M}^{\mathcal{D}}$ and $\boldsymbol{M}^{\mathcal{A}}$ data of a given group, the truncation number $p$ considered during the FMM assembly stage is determined by its closest far group, $p=p_{\max }$. For the $\boldsymbol{M}^{\mathcal{T}}$ data, the truncation number $p$ is determined by the two groups $\Gamma_{s}$ and $\Gamma_{o}$ involved in the translation, $p=p_{s o}$. During the iterative process, the aggregation step is carried out with $p=p_{\max }$, while $p=p_{\text {so }}$ suffices for the translation and disaggregation.

\section{Electrical Coupling of Saturated Hybrid Models}

The considered electrical circuit comprises a number of stranded conductors (e.g. coils) in the FE domain and a number of lumped components, viz voltage sources, resistive components and inductive components. By introducing loop currents associated with a set of independent current loops in the electrical circuit, Kirchhoff's current law automatically holds. Adding the electrical circuit equations to the hybrid FE-BE system (8), the coupled system of algebraic and differential equations can be written as (Lombard, 1993):

$$
\left[\begin{array}{ccc}
\boldsymbol{S} & \boldsymbol{C} & \boldsymbol{K} \\
\boldsymbol{D}^{T} & \boldsymbol{M} & 0 \\
0 & 0 & \boldsymbol{R}
\end{array}\right]\left[\begin{array}{c}
\boldsymbol{A} \\
\boldsymbol{Q} \\
\boldsymbol{I}_{l}
\end{array}\right]+\left[\begin{array}{ccc}
\boldsymbol{T} & 0 & 0 \\
0 & 0 & 0 \\
\boldsymbol{K}^{* T} & 0 & \boldsymbol{L}
\end{array}\right] \frac{\mathrm{d}}{\mathrm{d} t}\left[\begin{array}{c}
\boldsymbol{A} \\
\boldsymbol{Q} \\
\boldsymbol{I}_{l}
\end{array}\right]=\left[\begin{array}{c}
\boldsymbol{J}_{p m} \\
0 \\
\boldsymbol{U}
\end{array}\right]
$$

where $\boldsymbol{R}$ and $\boldsymbol{L}$ are square matrices that represent the resistive and inductive components respectively, $\boldsymbol{I}_{l}$ and $\boldsymbol{U}$ are vectors that contain the loop currents and the voltage sources respectively, $\boldsymbol{T}$ accounts for 
the eddy currents in conducting parts of the FE domain, $\boldsymbol{K}$ and $\boldsymbol{K}^{*}$ are due to the flux linkage of the conductors in the 2D FE model and $\boldsymbol{J}_{p m}$ contains the equivalent nodal currents due to the permanent magnets. The end-winding inductance of the FE conductors can be taken into account by means of lumped elements in the electrical circuit.

For a given voltage excitation and given initial conditions, the system (25) can be solved in the time domain. The time discretisation is commonly performed with the so-called $\theta$-method, which amounts to Crank-Nicholson method if $\theta=1 / 2$, and to the backward Euler method if $\theta=1$.

When saturation is included in the analysis, time stepping the system of algebraic and differential equations (25) produces a system of nonlinear equations for each time step. These nonlinear systems can be easily solved by means of the Newton Raphson (NR) method. The NR method linearises the nonlinear systems and an iterative method, e.g. GMRES, is applied.

In case of FMM, for every NR iteration the multiplications of $\boldsymbol{M}^{f a r}$ and $\boldsymbol{C}^{f a r}$ by the solution vector of the previous iteration $\boldsymbol{Q}$ are sped up in order to build the residual. Then for every GMRES iteration, the multiplications of $\boldsymbol{M}^{f a r}$ and $\boldsymbol{C}^{f a r}$ by a trial vector $\Delta \boldsymbol{Q}$ are also accelerated.

\section{Analysis of Electromechanical Devices}

We consider a rigid part that moves inside an airgap (as in the application example below).

Some elements of the complete system matrix are time dependent due to the movement (BE part) and magnetic saturation (FE part). These elements must be recalculated for any new time step. Furthermore, for every time step, the nonlinear contributions vary within the NR iterative loop. In order to reduce computation time, the whole system matrix is thus split up in three separate matrices. The contributions that remain constant throughout the simulation are calculated and stored. Those that depend only on the movement are saved in a matrix for every time step. Finally, those that are due to the nonlinear materials are reevaluated for every time step and every NR iteration.

\section{Mechanical equation}

In the FE-BE simulation, the position of the mover is either a given function of time or follows from the magnetic and other forces exerted on it. In the latter case, the mechanical equation has to be considered alongside the electromagnetic equations (25). If the movement is purely translational, it reads:

$$
m \frac{\mathrm{d} v(t)}{\mathrm{d} t}+\xi v(t)+k x(t)=F(t), \quad v(t)=\frac{\mathrm{d} x(t)}{\mathrm{d} t}
$$

where $x(t)$ is the position, $v(t)$ the speed, $m$ the mass, $\xi$ the viscous friction coefficient, $k$ the elastic constant and $F(t)$ the total applied force including the magnetic forces. We adopt the so-called "weak electromechanical coupling", i.e. the electromagnetic and mechanical equations are solved alternatively.

\section{Computation of the magnetic force}

The magnetic force $\underline{F}$ exerted on the moving part can be calculated by integrating the Maxwell stress tensor $\underline{\underline{T}}$ along a contour enclosing the moving part. If the FE domain $\Omega$ comprises an air layer around the moving part, the method proposed in (Henrotte, 2000) can be used as well. It amounts to a surface integral on the air layer $S$ :

$$
\underline{F}=l_{z} \int_{S} \frac{1}{\delta} \underline{\underline{T}} \underline{n} \mathrm{~d} s \quad \text { with } \quad \underline{\underline{\mathrm{T}}}=\frac{1}{\mu_{0}}\left(\underline{\mathrm{b}} \underline{\mathrm{b}}-\frac{\mathrm{b}^{2}}{2} \underline{\underline{1}}\right),
$$

where $l_{z}$ is the axial length of the device, $\delta$ the local thickness of the FE air layer and $\underline{n}$ is the outward unitary normal vector. This air layer also allows to avoid the oscillation problem that may occur if part of $\Gamma$ coincides with an air-iron interface (Henrotte, 2000; Geuzaine, 2001). 


\section{FMM taking movement into account}

The decomposition in groups $\Gamma=\bigcup_{g=1}^{\# g} \Gamma_{g}$ is preserved during movement. Expressing the ratio of the new complex distance between an element in a group and its group center (either observation or source) to the previous one as:

$$
\frac{\boldsymbol{z}_{o c}^{\prime}-\boldsymbol{z}_{o}^{\prime}}{\boldsymbol{z}_{o c}-\boldsymbol{z}_{o}}=e^{\imath \Delta \phi_{o}}, \quad \frac{\boldsymbol{z}_{s}^{\prime}-\boldsymbol{z}_{s c}^{\prime}}{\boldsymbol{z}_{s}-\boldsymbol{z}_{s c}}=e^{\imath \Delta \phi_{s}},
$$

and the ratio of the new complex distance between two group centers to the previous one as:

$$
\frac{\boldsymbol{z}_{o c}^{\prime}-\boldsymbol{z}_{s c}^{\prime}}{\boldsymbol{z}_{o c}-\boldsymbol{z}_{s c}}=\frac{r_{c}^{\prime}}{r_{c}} e^{\imath \Delta \phi_{o s}}
$$

where the prime indicates the new positions after motion, it is easy to find the relations between the previous and the new FMM data. Indeed, from (21-23), it follows:

$$
\begin{array}{r}
\boldsymbol{M}_{o, k, u}^{\prime \mathcal{D}}=\boldsymbol{M}_{o, k, u}^{\mathcal{D}} e^{\imath u \Delta \phi_{o}}, \quad \boldsymbol{M}_{s, l, v}^{\prime \mathcal{A}}=\boldsymbol{M}_{s, l, v}^{\mathcal{A}} e^{\imath u \Delta \phi_{s}} \\
\quad \text { and } \quad \boldsymbol{M}_{u, v}^{\prime \mathcal{T}}=\boldsymbol{M}_{u, v}^{\mathcal{T}}\left(\frac{r_{c}}{r_{c}^{\prime}}\right)^{u+v} e^{\imath(u+v) \Delta \phi_{o s}}
\end{array}
$$

This way, the integrals (21-23) do not have to be reevaluated. For updating the disaggregation, aggregation and translation data, a product of the previous data with the corresponding exponential factor suffices. In the particular case of purely translational movement, $\Delta \phi_{o}=\Delta \phi_{s}=0$, the disaggregation and aggregation data do not vary. Only the translation data have to be modified.

\section{Application Example}

The methods briefly outlined above are now applied to a 2D model of a linear actuator (Henrotte, 2000). As shown in Figure 3, the actuator comprises a yoke, two permanent magnets, two coils and a mover. The airgaps above and below vary, depending on the position of the mover, between $0.3 \mathrm{~mm}$ and $15.7 \mathrm{~mm}$. The two lateral gaps equal $0.5 \mathrm{~mm}$. The permanent magnets have a constant horizontal remanent induction of $b_{r}=0.8 \mathrm{~T}$ and a relative permeability of $\mu_{r}=1.03$. The yoke and the mover are made of iron. Eddy currents in the magnets and in the laminated yoke and mover are neglected.

The permanent magnets constitute a magnetic lock that keeps the mover either in the upper or lower position tending to diminish the residual airgap. The mover is moved down or up by applying a voltage pulse to one of the coils. The commutation is facilitated by two springs. The vertical force they exert on the mover as well as the force due to the magnets are shown in Figure 4 as a function of position.

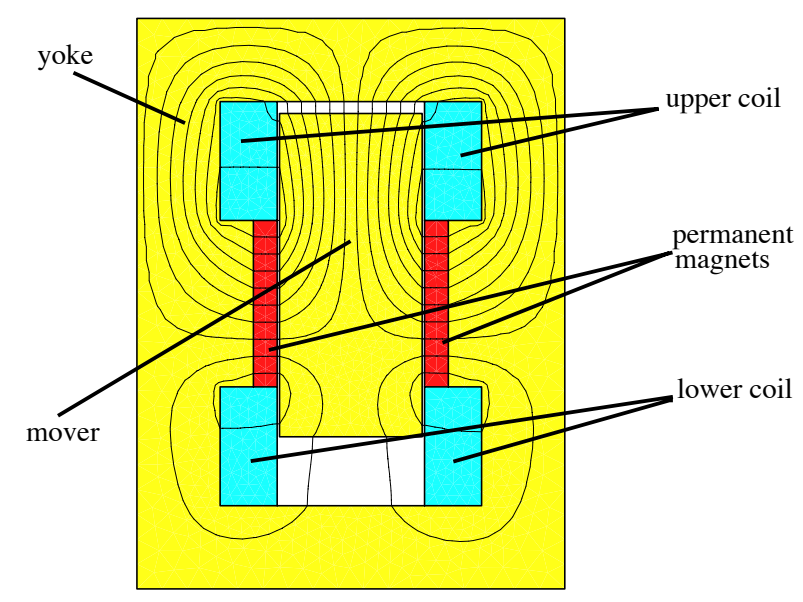

Figure 3. 2D model of a linear actuator 
Both linear and nonlinear dynamic simulations are performed. For the linear analysis, the relative permeability of the iron is taken as $\mu_{r}=1000$. For the nonlinear study, a nonlinear single-valued $\mathrm{BH}$ curve is adopted and the iterative NR method is applied for every time step. The computational cost for both the nonaccelerated and the accelerated hybrid model will be discussed.

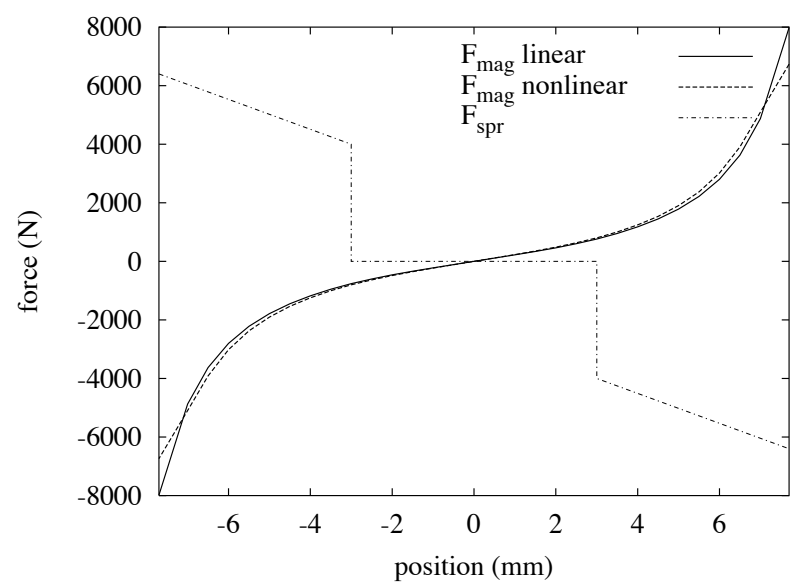

Figure 4. Magnetic force due to the permanent magnets and the spring force in function of the position of the mover. The mover is in the middle of the gap when $x=0$

The FE domain $\Omega$ comprises the yoke, the mover, the permanent magnets and the coils. On its outer boundary, the Dirichlet condition $a=0$ is imposed. In order to compute the magnetic force as mentioned in the previous section, an FE air layer of uniform thickness $\delta=0.2 \mathrm{~mm}$ is placed around the mover.

The BE domain is constituted by the outer contour of the air layer enclosing the moving piece, denoted $\Gamma_{1}$, and the outer boundary of the airgap, denoted $\Gamma_{2}$. The hybrid FE-BE discretisation, with 4083 triangular elements and 264 straight line segments, yields 2372 unknowns for the electromagnetic analysis: 2108 for $a$ and 264 for $q$. We adopt piecewise constant basis functions for $q$ and evaluate the integrals (10) and (12) analytically.

When the single-level FMM is applied to speed up the BE part of the hybrid model, the contour $\Gamma_{1}$ (134 segments) and contour $\Gamma_{2}$ (130 segments) are split up in 26 and 21 groups respectively. This distribution was found to be optimal: using more groups leads to a higher computation time for solving the system of equations while using less groups increases the assembly time. Two groups (on either $\Gamma_{1}$ or $\Gamma_{2}$ ) are considered to be far groups if there are at least two groups in between. This corresponds to $R_{s} / d \leq 1 / 6$ and $R_{o} / d \leq 1 / 6$, or, according to Figure 2 , to a maximum truncation number $p_{\max }=8$ for $\varepsilon=10^{-6}$. The classical law $p=\log _{2}(1 / \varepsilon)$ leads to a truncation number of 20 .

The voltage supply is included in the model by coupling a simple electrical circuit with the FE part of the hybrid model.

The system of algebraic and differential equations is time-stepped using the backward Euler scheme. A time interval of $50 \mathrm{~ms}$ (sufficiently long for achieving the commutation) has been studied with $\Delta t=$ $0.4 \mathrm{~ms}$ (125 time steps). The mechanical equation and the electromagnetic system are solved alternatively obtaining the new position and the new magnetic force respectively. A magnetostatic calculation with only the permanent magnet excitation is carried out to supply the correct initial value for the dynamic simulation.

\section{Calculation results}

At $t=0$, the mover is in the upper position $(x=7.7 \mathrm{~mm})$ and a $15 \mathrm{~V} 10 \mathrm{~ms}$ voltage pulse is applied to the lower coil. The time evolution of the current in the lower coil for both the linear and nonlinear analysis is depicted in Figure 5. The case without movement is also shown. In the latter case, the coil 
behaves as an RL circuit and an exponential evolution of the current is expected and also observed. The movement affects considerably the time variation of the current.

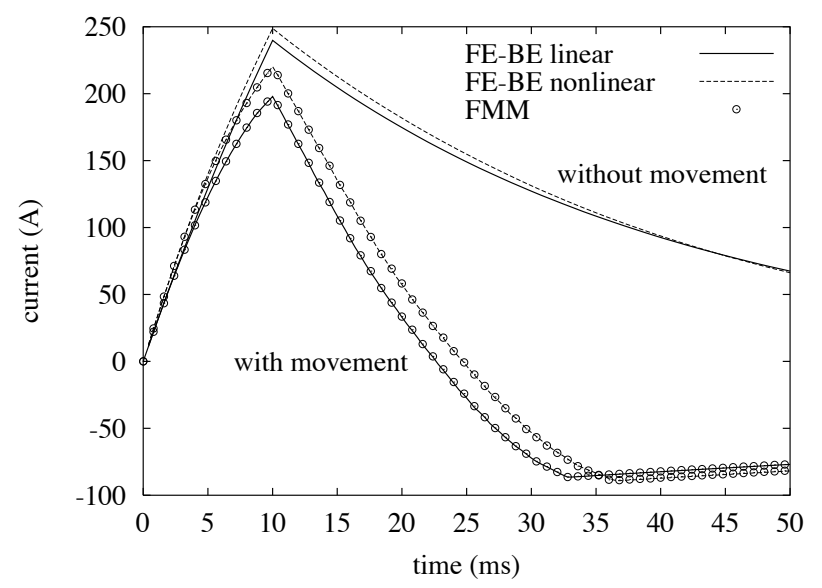

Figure 5. Evolution with time of the current in the lower coil when a $15 \mathrm{~V} 10 \mathrm{~ms}$ voltage pulse is applied: without and with movement

Figures 6 and 7 show the position and speed of the mover in function of time. The mover reaches the lower position after $33 \mathrm{~ms}$ and $36 \mathrm{~ms}$ in the linear and nonlinear analysis respectively.

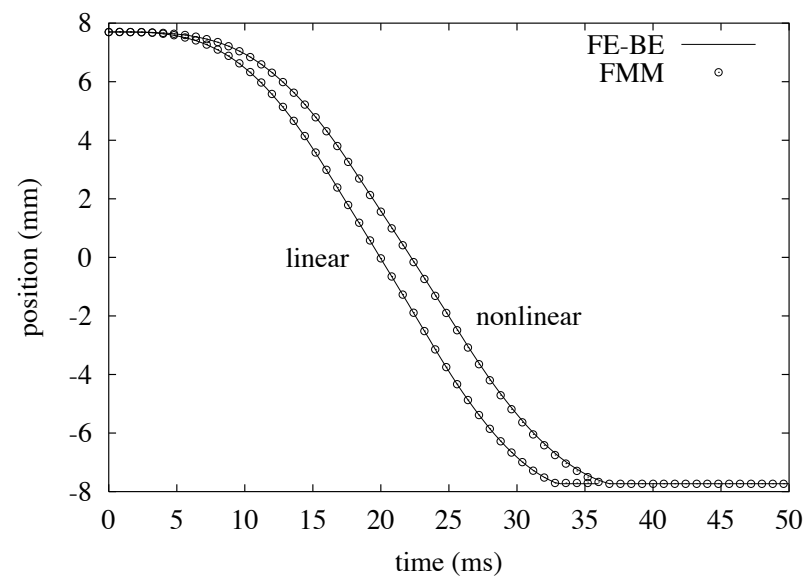

Figure 6. Position ( $\mathrm{mm}$ ) of the mover in function of time (ms) for the linear and nonlinear analysis with the FE-BE method accelerated or not by the FMM

All results obtained with the accelerated FE-BE method present an excellent agreement with those of the nonaccelerated FE-BE.

\section{Computational cost}

All computations have been carried out on a 400 MHz MIPS R12000 Processor. The approximate calculations times supplied hereafter give an indication of computational efficiency of the FMM accelerated FE-BE method compared to the nonaccelerated FE-BE method.

The systems of algebraic equations are solved by means of the iterative solver GMRES (Saad, 1986) with ILU-preconditioning. In case of FMM acceleration, the preconditioning is based on the sparse matrix comprising the complete FE contribution but only the BE near-field interactions. For the 2D problem at hand, this results in an increase of the number of GMRES iterations for solving the linear or linearised systems of equations, disadvantage which is largely outweighed by the fact that $\boldsymbol{M}^{\text {far }}$ and $C^{f a r}$ are not evaluated explicitly. 


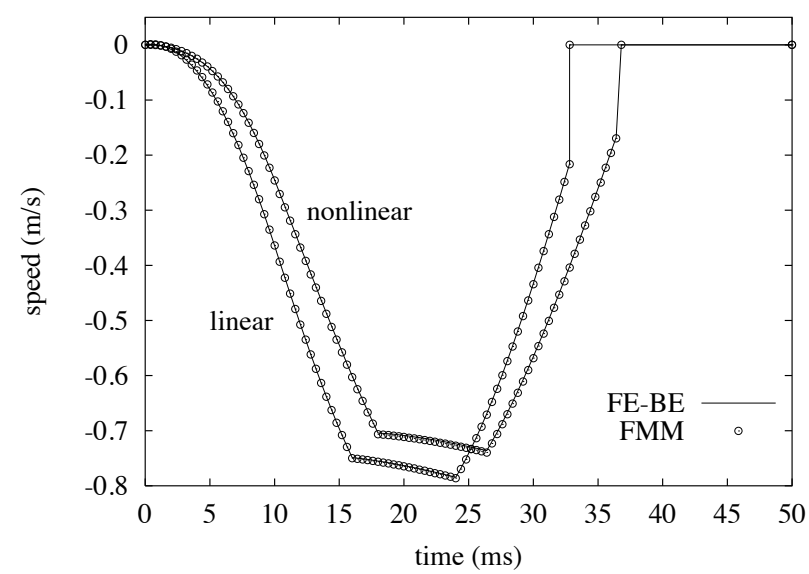

Figure 7. Speed $(\mathrm{m} / \mathrm{s})$ of the mover in function of time $(\mathrm{ms})$ for the linear and nonlinear analysis with the FE-BE method accelerated or not by the FMM

Indeed, the linear calculation takes $1420 \mathrm{~s}$ without FMM and $470 \mathrm{~s}$ with FMM acceleration. For the nonlinear simulation, with 3 to $5 \mathrm{NR}$ iterations per time step, the computation times are $2266 \mathrm{~s}$ without FMM acceleration and $990 \mathrm{~s}$ with the FMM acceleration. The reduction in computation time achieved by the FMM in the linear and nonlinear case is thus $70 \%$ and $56 \%$ respectively.

\section{Conclusion}

The resolution of a $2 \mathrm{D}$ electromechanical problem by means of a hybrid FE-BE model accelerated with the FMM has been elaborated. A 2D linear actuator has been modelled taking into account saturation, the electrical circuit coupling and the mechanical equation. An excellent agreement between the results obtained with the nonaccelerated and the accelerated FE-BE method has been achieved. Updating the acceleration (aggregation, disaggregation and translation) data for every new position of the mover has proved to be simple and computationally cheap. Applying FMM accelerated FE-BE method yields significantly savings in computation time. A higher speed is envisaged when applying a multilevel FMM scheme.

\section{Acknowledgement}

The research was carried out in the frame of the Inter-University Attraction Poles for fundamental research funded by the Belgian State. P. Dular and C. Geuzaine are Research Associate and Postdoctoral Researcher respectively with the Belgian Fund for Scientific Research (F.N.R.S.).

\section{References}

Buchau, A., Huber, C. J., Rieger W. and Rucker W. M. (2000), "Fast BEM computations with the adaptive multilevel fast multipole method", IEEE Transactions on Magnetics, Vol. 36, No. 4, July, pp. 680-684.

Burkholder R. J. and Kwon, D.-H. (1996), "High-frequency asymptotic acceleration of the fast multipole method", Radio Science, Vol. 31, No. 5, May, pp. 1199-1206.

Fetzer, J., Kurz, S., Lehner G. and Rucker, W. M. (1999), "Analysis of an actuator with eddy currents and iron saturation: Comparison between a FEM and a BEM-FEM coupling approach", IEEE Transactions on Magnetics, Vol. 35, No. 3, May, pp. 1793-1796.

Geuzaine, C., Tarhasaari, T., Kettunen, L. and Dular, P. (2001), "Discretisation schemes for hybrid methods", IEEE Transactions on Magnetics, Vol. 37, No. 5, September, pp. 3112-3115.

Henrotte, F., Nicolet, A., Hédia, H., Genon, A. and Legros W. (1994), "Modelling of electromechanical relays taking into account movement and electric circuits", IEEE Transactions on Magnetics, Vol. 30, No. 5, September, pp. 3236-3239. 
Henrotte, F. (2000), Modelling of electromagnetic forces and their effects on arbitrary structures (in French), Ph.D. Thesis 201, Faculty of Applied Sciences, University of Liège, Belgium.

Lombart, P. and Meunier, G. (1993), "A general purpose method for electric and magnetic combined problems for 2D, axisymmetric and transient systems", IEEE Transactions on Magnetics, Vol. 29, No. 2, March, pp. $1737-1740$.

Lu N. and Jin J.-M. (1996), "Application of fast multipole method to finite-element boundary-integral solution of scattering problems", IEEE Transactions on Antennas and Propagation, Vol. 44, No. 6, June, pp. 781-786.

Nabors, K. and White, J. (1991), "FastCap: A multipole accelerated 3-D capacitance extraction program", IEEE Transactions on Computer-Aided Design, Vol. 10, No. 11, November, pp. 1447-1459.

Nysveen, A. and Nilssen, R. (1997), "Time domain simulation of magnetic systems with a general moving geometry", IEEE Transactions on Magnetics, Vol. 33, No. 2, March, pp. 1394-1397.

Rokhlin, V. (1983), "Rapid solution of integral equations of classical potential theory", Journal of Computational Physics, Vol. 60, pp. 187-207.

Saad, Y. and Schultz, M. H. (1986), "GMRES: A Generalized Minimal Residual Algorithm for solving nonsymmetric linear systems", SIAM J. Sci. Comput., Vol. 7, No. 3, July, pp. 856-869.

Sabariego, R. V., Gyselinck, J., Geuzaine C., Dular, P. and Legros W. (2002), "Application of the fast multipole method to hybrid finite element-boundary element models", Proceedings of the Second International Conference on Advanced Computational Methods in Engineering (ACOMEN), Liège (Belgium), May 28-31.

Song, J. M. and Chew, W. C. (1995), "Multilevel fast-multipole algorithm for solving combined field integral equations of electromagnetic scattering", Micr. Opt. Tech. Lett., No. 1, September, pp. 14-19. 